\title{
Examine the Behavior of Node Mobility in ZigBee Sensor Network
}

\author{
Sandeep Kaur \\ M.Tech Scholar ,AIET \\ Faridkot, Punjab
}

\author{
Harinderpal Singh \\ Assistant Professor,AIET \\ Faridkot, Punjab
}

\author{
Gurjeevan Singh \\ DIC ECE , Poly-Wing, SBSSTC \\ Ferozepur, Punjab
}

\begin{abstract}
This paper is mainly based upon mobility. In this paper three topologies are used i.e. mesh, tree and hybrid topology. The hybrid topology is implemented by using the combination of mesh and tree topology. The data-based setup is done by changingmobile percentageof nodes. The performance of these topologies is measured using parameters throughput, MAC delay, MTR and MTS.The result show better performances of hybrid topology, which provides low MAC delay and effective throughput in the mobile network. The simulation is done by OPNET modeler 14.5.
\end{abstract}

\section{Keywords}

IEEE 802.15.4, ZigBee, OPNET Modeler, Throughput, MAC Delay.

\section{INTRODUCTION}

There are three short-range wireless three protocols: the Bluetooth, ZigBee, and Wi-Fi, are corresponding to the IEEE 802.15.1, 802.15.4, and $802.11 \mathrm{a} / \mathrm{b} / \mathrm{g}$ standards, respectively[10]. ZigBee was conceived in 1998, standardized in 2003 and revised in 2006. ZigBee is based on the IEEE 802.15.4 standard along with other protocols like Wi-Fi and bluetooth. It was developed by IEEE 802.15.4 Task Group and ZigBee Alliance. IEEE 802.15.4 protocol stack defines two layer; MAC layer and Physical layer. The MAC layer send long data frames over radios channels with specified modulation and spreading techniques. The Physical layer is responsible for transmission and reception of data by providing a desired radio channel. It also provides modulation on outgoing signal and demodulation on incoming signals .The application layer provides the interface to users. A single node can support 240 applications [1]. ZigBee defines the two layers of OSI model i.e. application layer, network layer. The ZigBee Network Layer (NWK) is responsible for Network management procedures, security and routing. It also encloses the neighbour tables and the storage of related information. The ZigBee protocol defines three types of wireless nodes Personal Area Network Coordinator, full function device and reduced function device. ZigBee coordinator is intended for starting the network and choosing the key network parameters. ZigBee routers are devices capable of routing data. ZigBee end-devices have no routing capability - these devices rely on their parents, the coordinator or routers, to transmit/ route their packets. ZigBee can operate in three network topologies i.e. star, mesh and tree. In star topology there are multiple end devices and a single coordinator. The end devices communicate through the coordinator. In mesh and tree topology a message can be transmitted through multiple routes. If a particular router fails, then ZigBee selfhealing mechanism will allow the network to search for an alternate path for the message to be passed [7]. The standard was developed to meet the following principal need of low cost, ultralow power consumption, use of unlicensed radio bands, cheap and easy installation, flexible and extendable networks [4]. It attempts to provide a low-data rate, lowpower, and low-cost wireless networking on the device levelCommunication.In this paper, the performance of Tree and mesh topologies have been analysed by giving the mobility support and implement a new hybrid topology by using tree and mesh topology in the combine form in a single scale network.

\section{APPLICATIONS OF ZIGBEE SENSOR NETWORKS}

In this section, we introduce the major applications of ZigBee wireless sensor network.

\subsection{Underground Cable System}

There are many sensors existing in WSNs technologies, such as coaxial cable sensors, inductive sensors, acoustic emission techniques, fiber optic distributes sensor, to supervise and diagnose the underground cables. In the underground power lines, various failures occur in joints, terminations and maintenance should be provided according to system requirement [11].

\subsection{Animals and Vegetation Control System}

In the surviving power grid, trees and branches of trees and destructive animals cause serious problems like damaging cable and cause short circuit. These problems lead to memory loss and make system unreliable. WSNs accomplish and sense animals, vegetation help to recover the damage occur by these animals and vegetation [11].

\subsection{Precision Agriculture}

A WSNs application in agriculture produces high crop production arising information systems and is known as Precision Agriculture [3]

\subsection{Building Automation}

The wireless technologies like Radio Frequency, Infrared, Bluetooth and Wi-Fi are used for the building automation, many sensors like motion detectors, smoke, gas, Door, Window, water level, etc. are used to detect and monitor the different physical condition in home, office and in other buildings. These sensors sense and send the input data to the main location such as owner and alert him if any unwanted event is detected such as gas leakage, intrusion in the building, water overflow etc. Hence maintain the security of building [5].

\subsection{Towers and Poles Monitoring}

The component failures on poles or tower in the surviving power grid lead to power outages and higher healing costs. To maximize the performance of system and equipment life 
timely monitoring and control of towers and poles is essential [11].

\subsection{Home and Office Automation}

The Wireless technologies like ZigBee, RFID, and GSM are used for home and office automation. ZigBee technology is used to open and close door from inside the home/office, temperature monitoring, gas detection and light controlling [6].

\subsection{Patient Monitoring}

Patients are being monitored using wireless sensor network. System is used to monitor physiology parameters such as heart beat, blood pressure etc. [12].

\section{WIRELESS PROTOCOLS}

This section introduces the Bluetooth, UWB, ZigBee, and WiFi protocols, which Corresponds to the IEEE 802.15.1, $802.15 .3,802.15 .4$, and $802.11 \mathrm{a} / \mathrm{b} / \mathrm{g}$ standards, respectively.

\section{$3.1 \quad$ Wi-Fi}

Wireless fidelity (Wi-Fi) is based upon IEEE $802.11 \mathrm{a} / \mathrm{b} / \mathrm{g}$ standards for wireless local area networks (WLAN). It allows users to access the Internet at broadband speeds when connected to an access point (AP) or in ad hoc mode. The frequency band of Wi-Fi is $2.4 \mathrm{GHz} ; 5 \mathrm{GHz}$. The IEEE 802.11 architecture consists of several components that interact to provide a wireless LAN .The basic cell of an IEEE 802.11 LAN is called a basic service set (BSS), which is a set of mobile or fixed stations. If a station moves out of its BSS, it can no longer directly communicate with other members of the BSS [9].

\subsection{Bluetooth}

Bluetooth, also known as the IEEE 802.15.1 standard is based on a wireless radio system. Bluetooth is a new short-range wireless technology designed to enable wireless communication between diverse devices[8]. Two connectivity topologies are defined in Bluetooth: the piconet and scatternet. A piconet is a WPAN formed by a Bluetooth device serving as a master in the piconet and one or more Bluetooth devices serving as slaves. A scatternet is a collection of operational Bluetooth piconets overlapping in time and space[9]. Bluetooth technology uses frequency-hopping spreadspectrum (FHSS) communication in the $2.4-\mathrm{GHz}$ industrial, scientific, and medical (ISM) band, in which unlicensed devices are permitted to communicate in most countries of the world [8].

\subsection{ZigBee}

ZigBee is based upon IEEE 802.15.4 standard. ZigBee is intended for use in those applications that require low data rate, long battery life, and secure networking [1]. Two different device types can participate in ZigBee: a fullfunction device (FFD) and a reduced function device (RFD). The FFD can operate in three modes serving as a PAN coordinator, a coordinator, or a device. An FFD can talk to RFDs or other FFDs, while an RFD can talk only to an FFD[9]. ZigBee Applications include wireless light switches; electrical meters with in-home-displays, traffic management systems etc. [1].

\section{COMPARATIVE STUDY}

Table 1 summarizes the main differences among the three protocols. Each protocol is based on an IEEE standard. Wi-Fi provides a higher data rate than that of Bluetooth and ZigBee.
Table 1:Comparison of Bluetooth, ZigBee and Wi-Fi

Protocols

\begin{tabular}{|c|c|c|c|}
\hline Standard & Bluetooth & ZigBee & Wi-Fi \\
\hline $\begin{array}{l}\text { IEEE } \\
\text { Specification }\end{array}$ & 802.15 .1 & 802.15 .4 & $802.11 \mathrm{a} / \mathrm{b} / \mathrm{g}$ \\
\hline Data protection & 16 -bit CRC & $\begin{array}{ll}16 & \text {-bit } \\
\text { CRC } & \end{array}$ & $32-$ bit CRC \\
\hline $\begin{array}{l}\text { Power } \\
\text { Consumption }\end{array}$ & Medium & Very low & High \\
\hline $\begin{array}{l}\text { Transmission } \\
\text { range }\end{array}$ & $10 \mathrm{~m}$ & $10-100 \mathrm{~m}$ & $100 \mathrm{~m}$ \\
\hline Basic cell & Piconet & Star & BSS \\
\hline $\begin{array}{l}\text { Channel } \\
\text { bandwidth }\end{array}$ & $1 \mathrm{MHz}$ & $\begin{array}{ll}0.3 / 0.6 & \\
\mathrm{MHz} ; & 2 \\
\mathrm{MHz}\end{array}$ & $22 \mathrm{MHz}$ \\
\hline Authentication & Shared secret & $\begin{array}{l}\text { CBC-MAC } \\
\text { (ext. of } \\
\text { CCM) }\end{array}$ & WPA2 (802.11i) \\
\hline Spreading & FHSS & DSSS & $\begin{array}{ll}\text { DSSS, } & \text { CCK, } \\
\text { OFDM } & \end{array}$ \\
\hline $\begin{array}{l}\text { Networking } \\
\text { Topology }\end{array}$ & $\begin{array}{l}\text { Ad-hoc, very } \\
\text { small } \\
\text { networks }\end{array}$ & $\begin{array}{l}\text { Ad-hoc, } \\
\text { peer to } \\
\text { peer, star, } \\
\text { or mesh }\end{array}$ & Point to hub \\
\hline $\begin{array}{l}\text { Operating } \\
\text { Frequency }\end{array}$ & $2.4 \mathrm{GHz}$ & $\begin{array}{l}868 \mathrm{MHz} \\
\text { (Europe) } \\
900-928 \\
\mathrm{MHz} \text { (NA), } \\
2.4 \mathrm{GHz} \\
\text { (worldwide } \\
\text { ) } \\
\end{array}$ & 2.4 and $5 \mathrm{GHz}$ \\
\hline Complexity & High & Low & High \\
\hline Applications & $\begin{array}{l}\text { Wireless } \\
\text { connectivity } \\
\text { between } \\
\text { devices such } \\
\text { as phone etc. }\end{array}$ & $\begin{array}{l}\text { Industrial } \\
\text { automation, } \\
\text { Medical } \\
\text { monitoring, } \\
\text { Low-power } \\
\text { sensors etc. }\end{array}$ & $\begin{array}{l}\text { Wireless LAN } \\
\text { connectivity, } \\
\text { broadband } \\
\text { Internet access. }\end{array}$ \\
\hline
\end{tabular}

Sources[2][9][10].

\section{SIMULATION SETUP}

The simulation of ZigBee network has been done by using OPNET modeler 14.5.

Table 2: Simulation parameters

\begin{tabular}{|l|l|}
\hline Network Size & $45 \mathrm{~m} * 45 \mathrm{~m}$ \\
\hline Number of Nodes & 36 nodes \\
\hline Simulation Duration & $300 \mathrm{Sec}$ \\
\hline Mobility Model & Random Waypoint \\
\hline $\mathrm{Lm}$ & 5 \\
\hline $\mathrm{Cm}$ & 10 \\
\hline $\mathrm{Rm}$ & 10 \\
\hline
\end{tabular}


Using table 1, three scenarios has been designed in which percentage of mobile nodes varies from 20 to 80 percent and using random waypoint mobility model the speed of mobile nodes are $5 \mathrm{~m} / \mathrm{s}$. The nodes are randomly placed anywhere in the network. In the first scenario tree topology and in second scenario mesh topology is used. In third scenario the combination of tree and mesh topologies are used to implement a hybrid topology.

\section{RESULTS AND DISCUSSIONS}

The given result shows the performance of hybrid $(\mathrm{h})$, tree $(\mathrm{t})$, mesh (m) with $20 \%$ (1), 40\%(2), 60\%(3), 80\% (4) of mobile nodes in the network. Using followingparameters the performance of the network has been observed.

\subsection{Throughput}

Throughput is the ratio of the total amount of data that a receiver receives from a sender to a time it takes for receiver to get the last packet. Throughput is the data quantity transmitted correctly starting from the source to the destination within a specified time (seconds). Throughput is inversely proportional to the delay in network. Throughput is measured with various factors such as packet collisions, obstructions between nodes and the type of used topology. The results shown in Figures 1, 2, 3and 4 conclude that maximum throughput is achieved by tree topology and minimum throughput is achieved in mesh topology. Throughput varies in hybrid topology as mobile percentage of nodes increases.

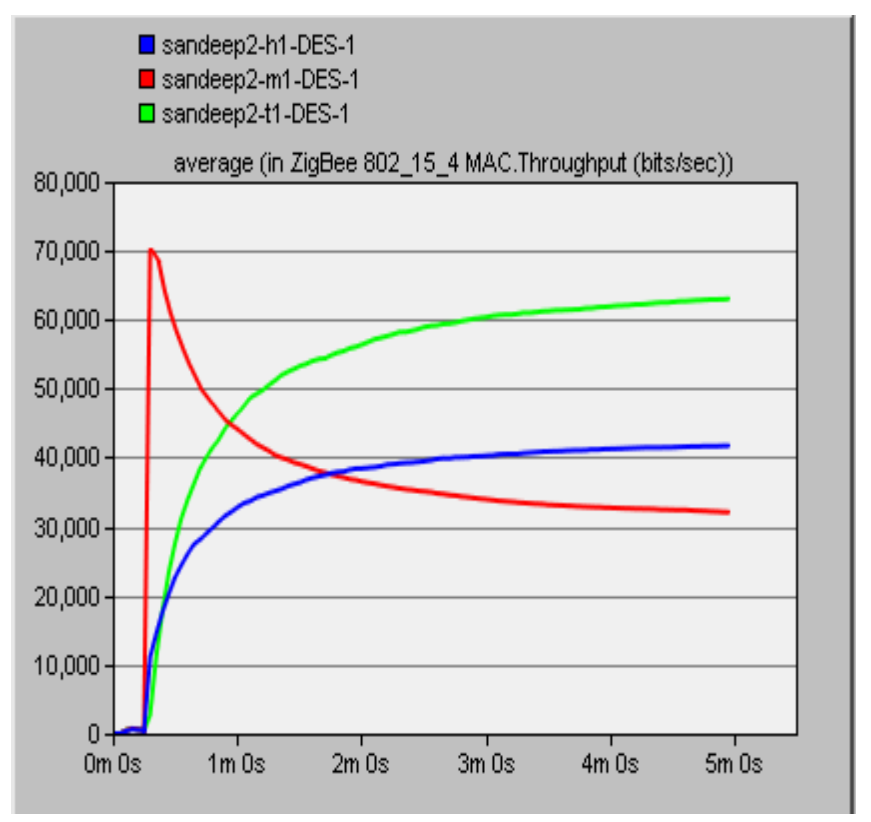

Figure 1Throughput of tree, mesh and hybrid topologies with $20 \%$ of mobile nodes

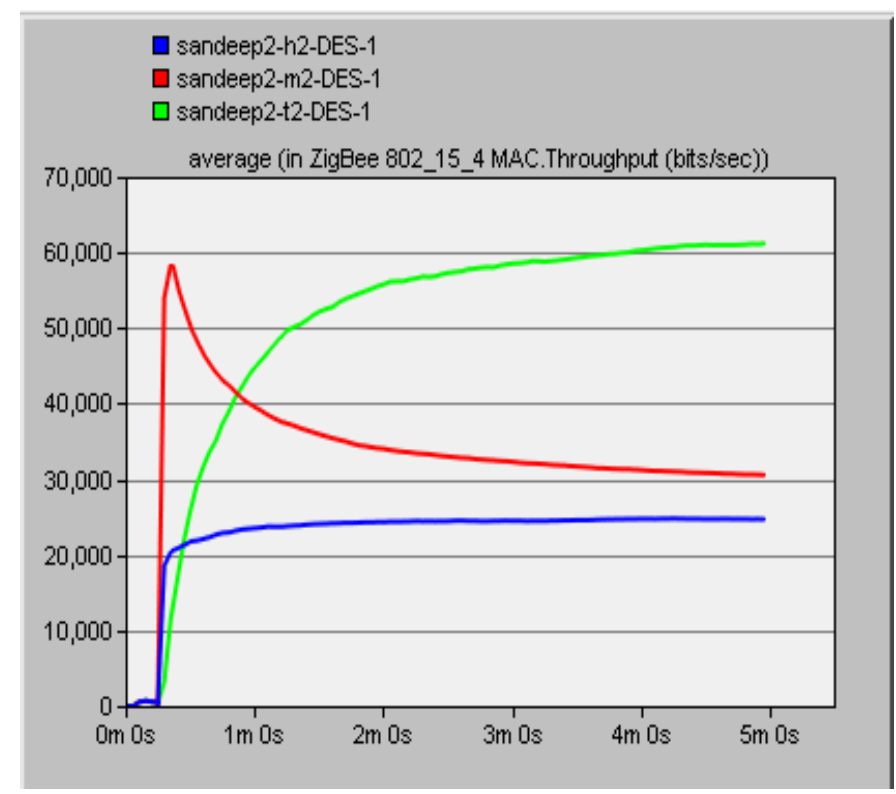

Figure 2 Throughput of tree, mesh and hybrid topologies with $40 \%$ of mobile nodes

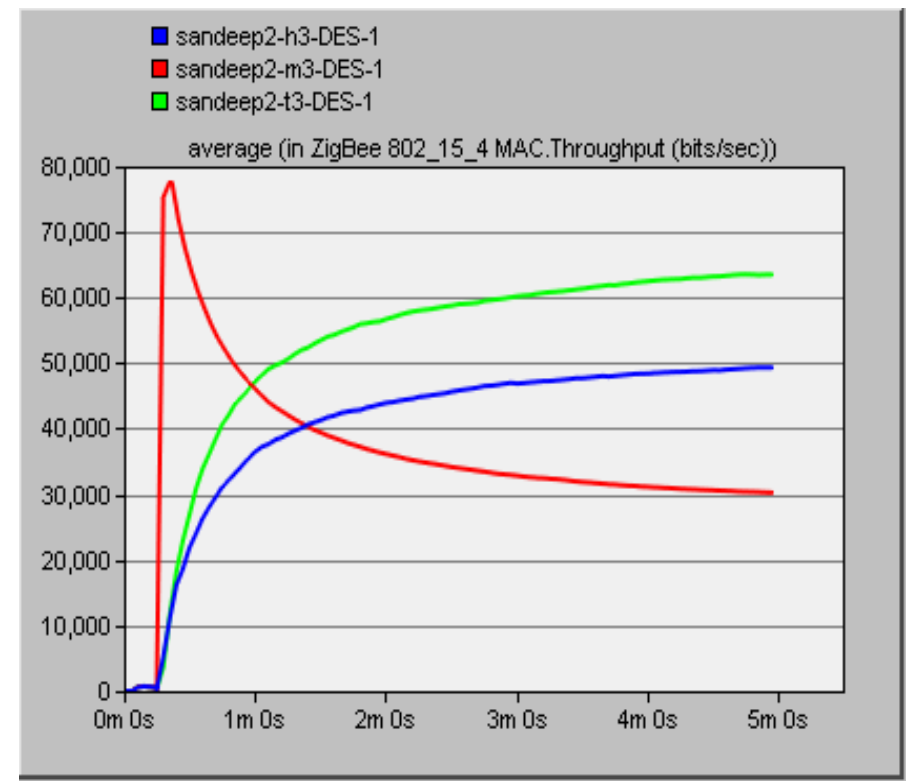

Figure3 Throughput of tree, mesh and hybrid topologies with $60 \%$ of mobile nodes 


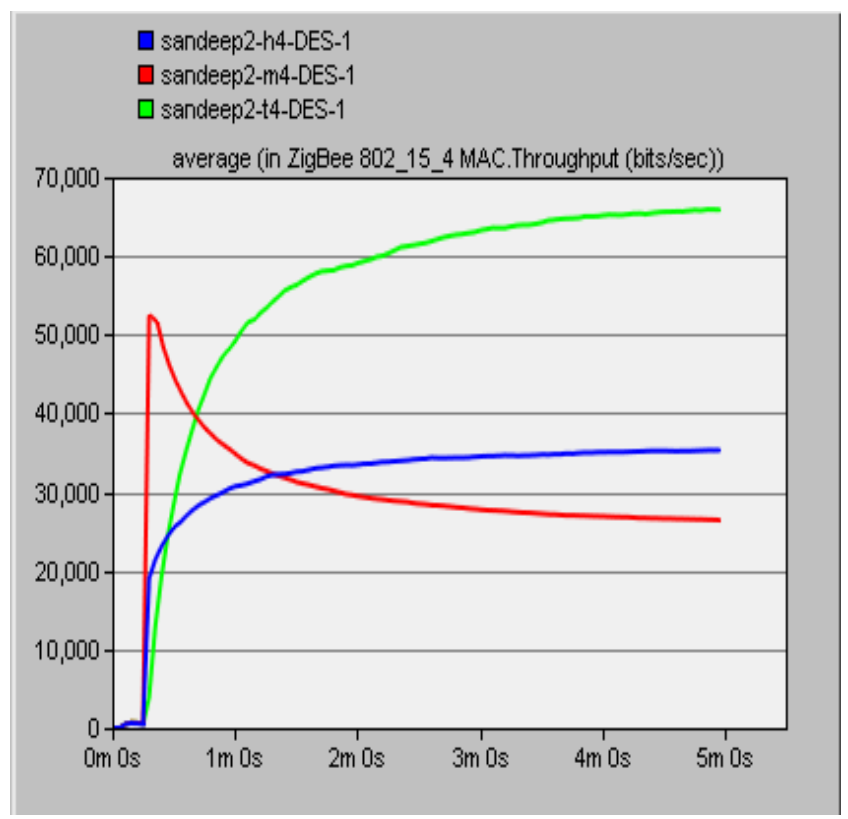

Figure 4 Throughput of tree, mesh and hybrid topologies with $80 \%$ of mobile nodes

\subsection{MAC Delay}

Represents the end to end delay of all the packets received by the 802.15.4 MACs of all WPAN nodes in the network and forwarded to the higher layer. Figures 5, 6, 7 and 8 shows that MAC delay of hybrid topology is minimumand MAC delay of tree topology is maximum in all simulation. The MAC delay in mesh topology rapidly increases at the start time duration and then fall down.

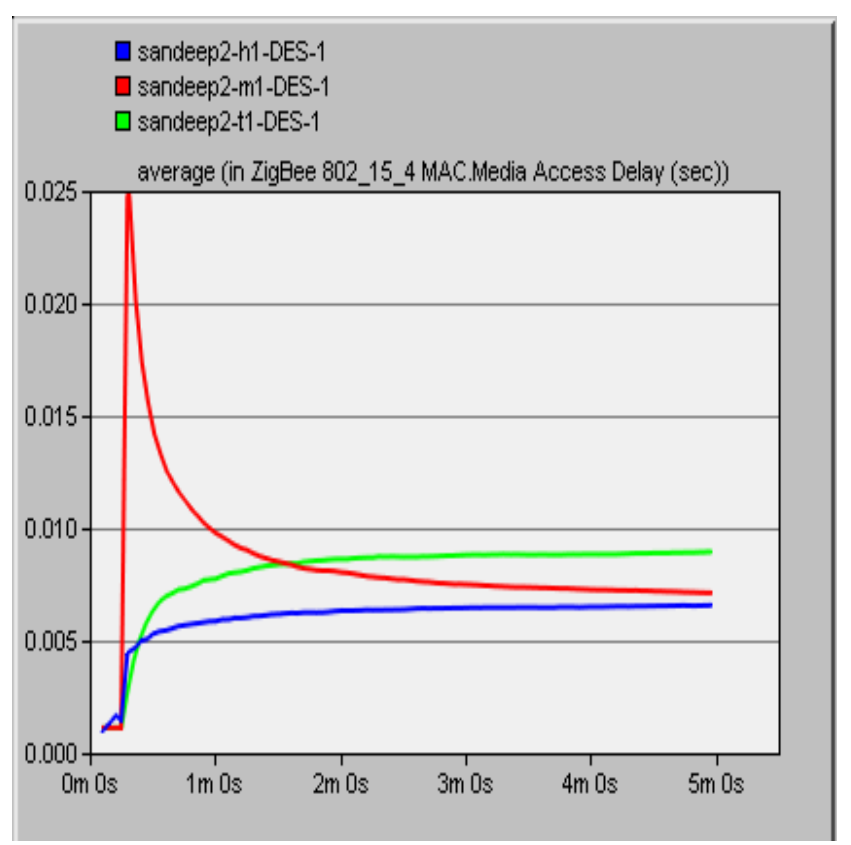

Figure 5 MAC delay of tree, mesh and hybrid topologies with $20 \%$ of mobile nodes

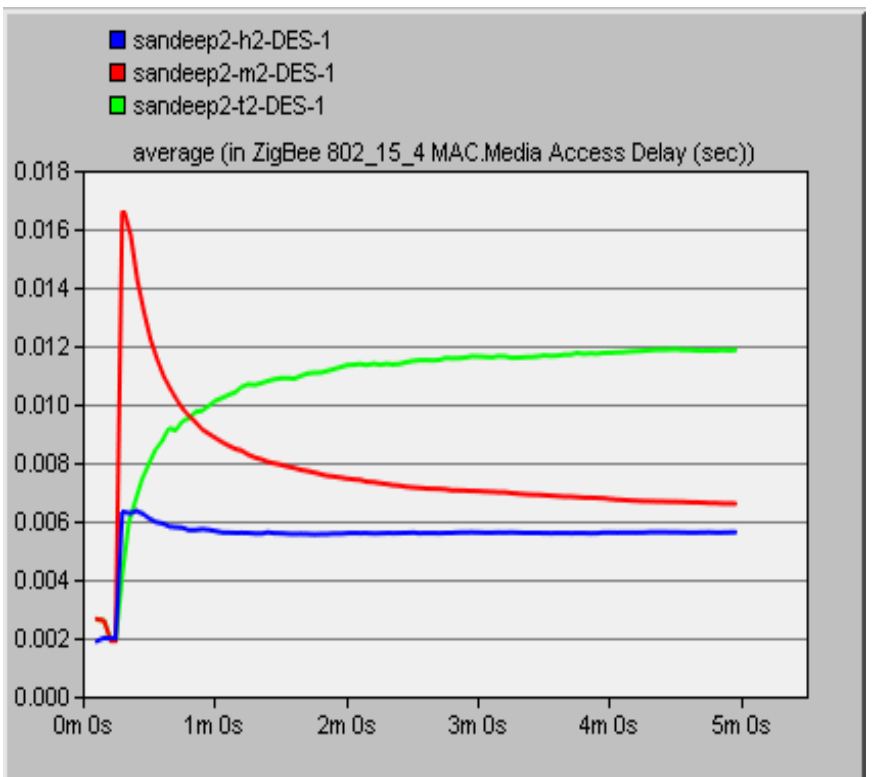

Figure 6 MAC delay of tree, mesh and hybrid topologies with $40 \%$ of mobile nodes

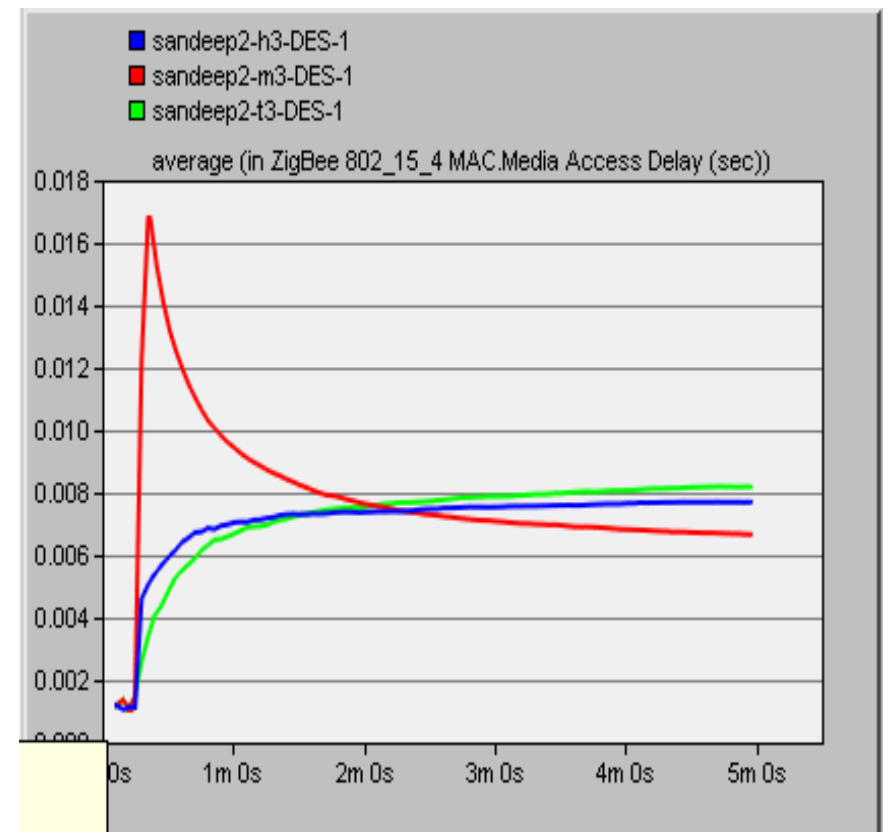

Figure 7 MAC delay of tree, mesh and hybrid topologies with $60 \%$ of mobile nodes 


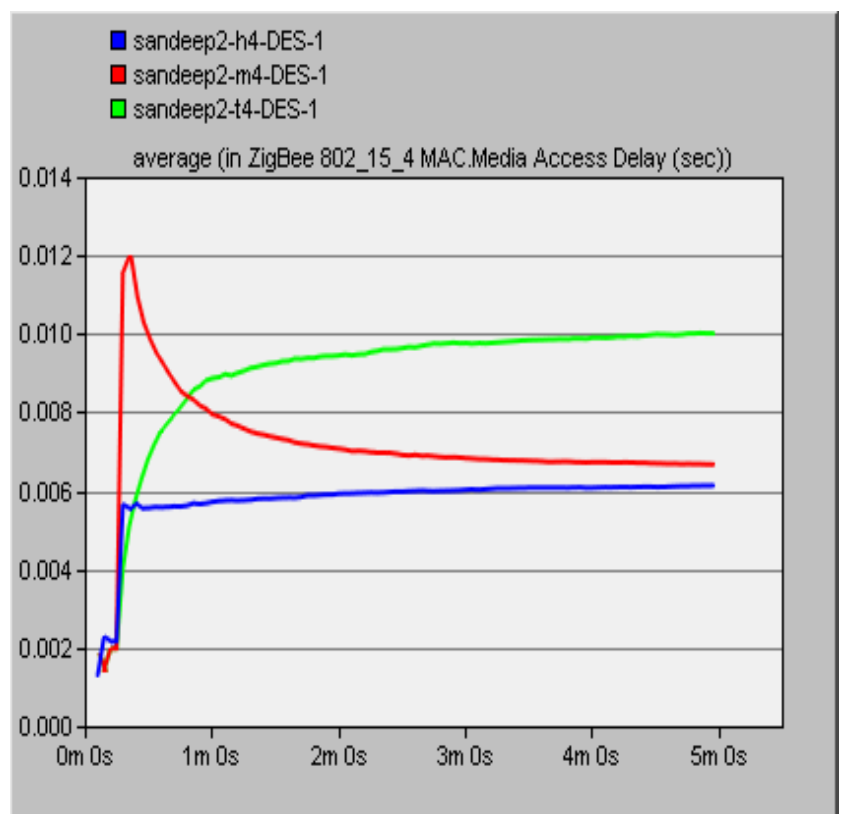

Figure 8 MAC delay of tree, mesh and hybrid topologies with $80 \%$ of mobile nodes

\subsection{Management Traffic Received}

(MTR) These statistics record the total amount of management traffic (i.e., beacon and Block-ACK frames) received from the physical layer on this network interface. These statistics record successful arrival of management frames even if they are not destined to this interface. When these statistics are reported in units of bits/second, the physical and the MAC header sizes are included in the computation of the total amount of traffic received. Figures 9, 10,11 and 12 shows that maximum MTR is achieved in mesh topology and minimum in case of tree topology. But in case of hybrid topology MTR increases in 40 and 80 percent of mobile nodes and decreases in 20 and 60 percent of mobile nodes.

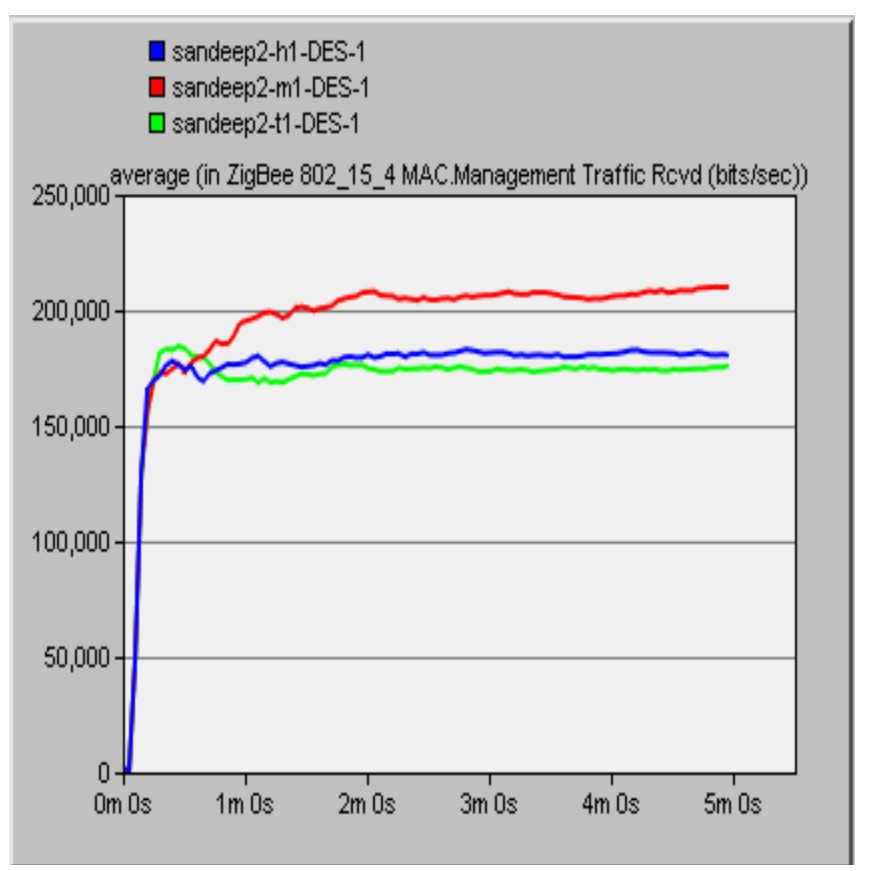

Figure 9 MTR of tree, mesh and hybrid topologies with $20 \%$ of mobile nodes

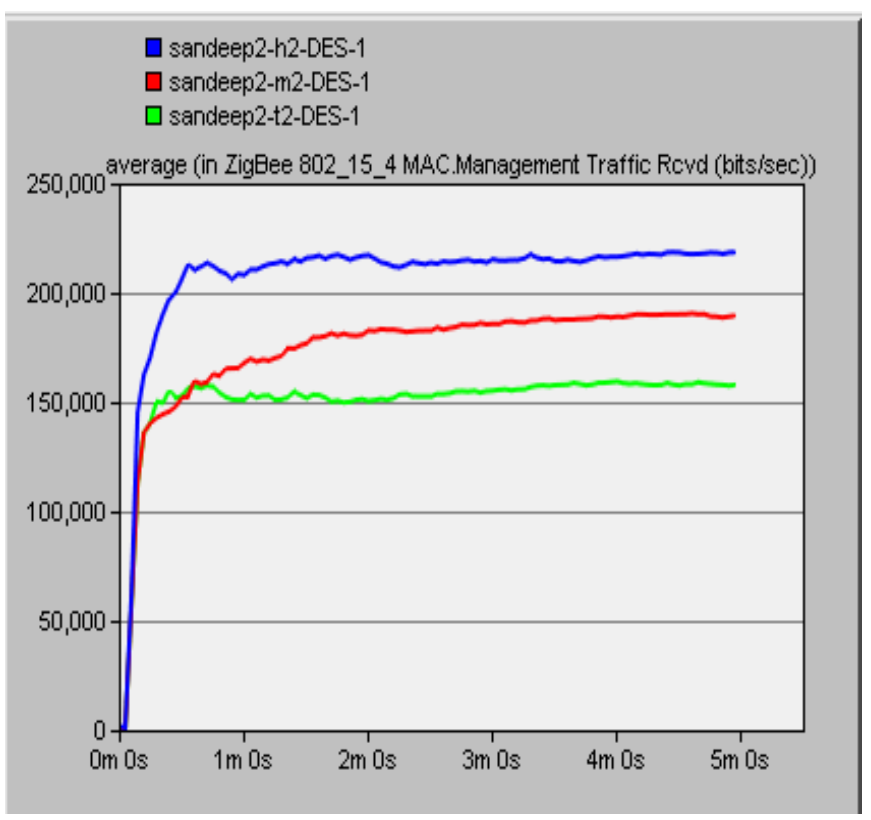

Figure10 MTR of tree, mesh and hybrid topologies with $40 \%$ of mobile nodes

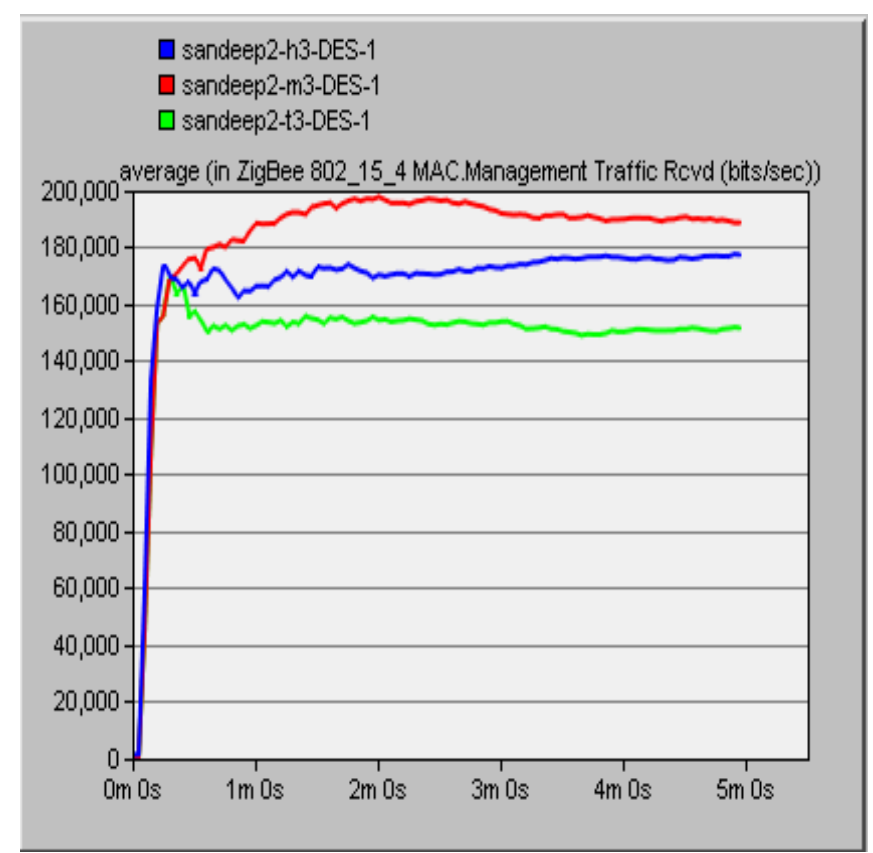

Figure 11 MTR of tree, mesh and hybrid topologies with $60 \%$ of mobile nodes 


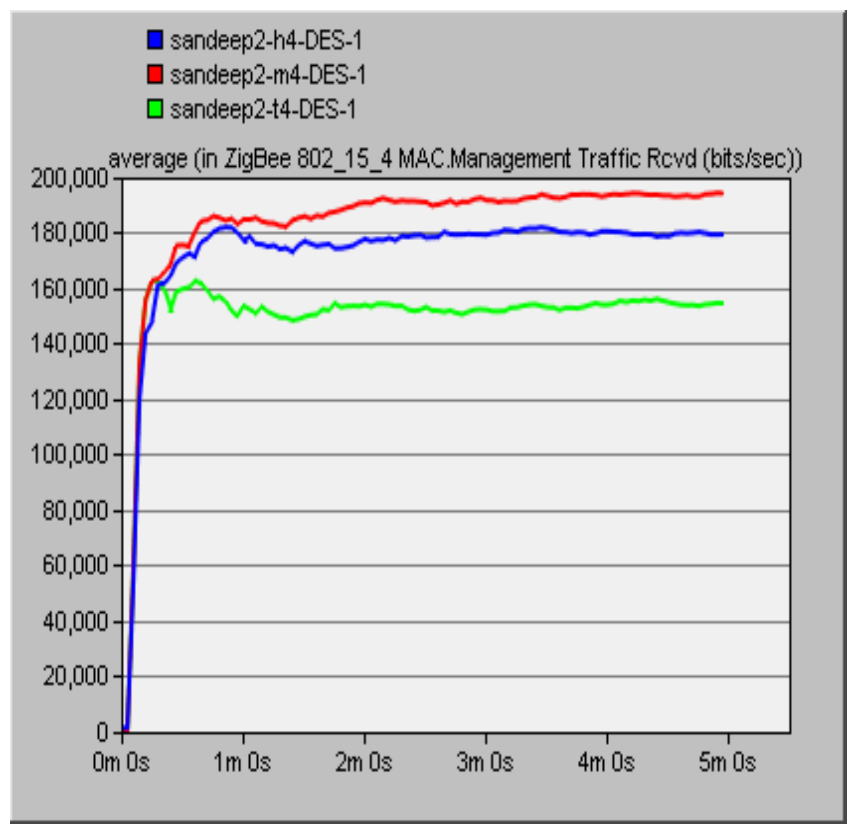

Figure12 MTR of tree, mesh and hybrid topologies with $80 \%$ of mobile nodes

\subsection{Management Traffic Send (MTS)}

These statistics record the total amount of management traffic (i.e., beacon and Block-ACK frames) transmitted by the network interface onto the physical medium. When these statistics are reported in units of bits/second, the physical and the MAC header sizes are included in the computation of the total amount of traffic sent. Figures 13, 14, 15 and 16 shows that maximum MTS is achieved in mesh topology and minimum MTS is achieved in tree topology but in hybrid topology it is almost same in all cases.

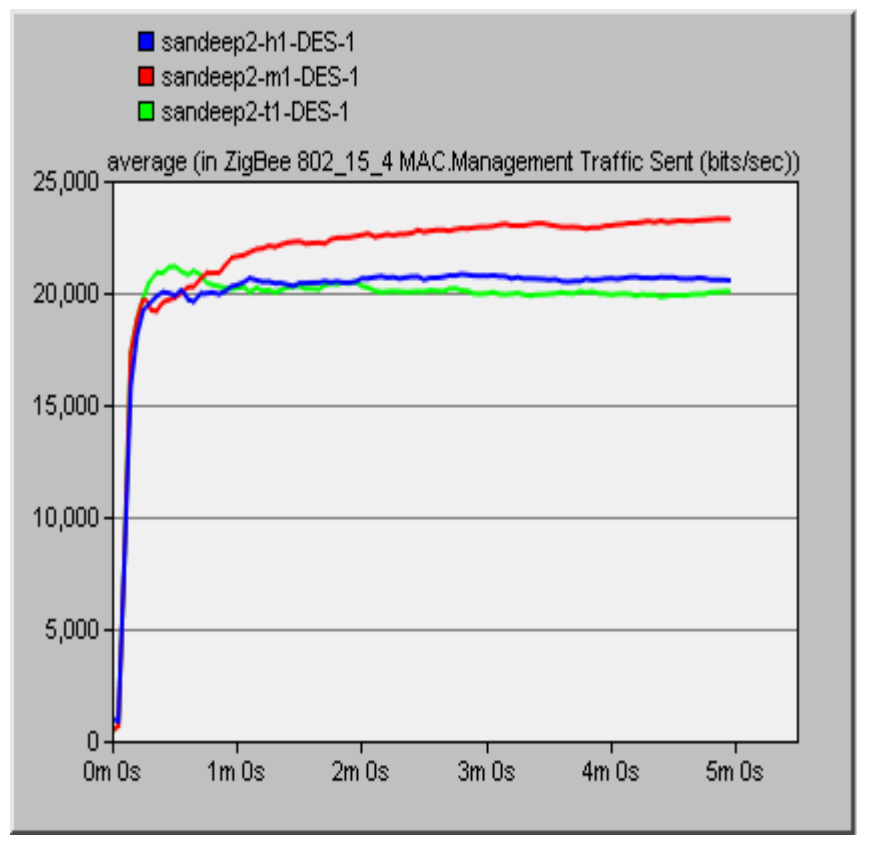

Figure13 MTS of tree, mesh and hybrid topologies with $20 \%$ of mobile nodes

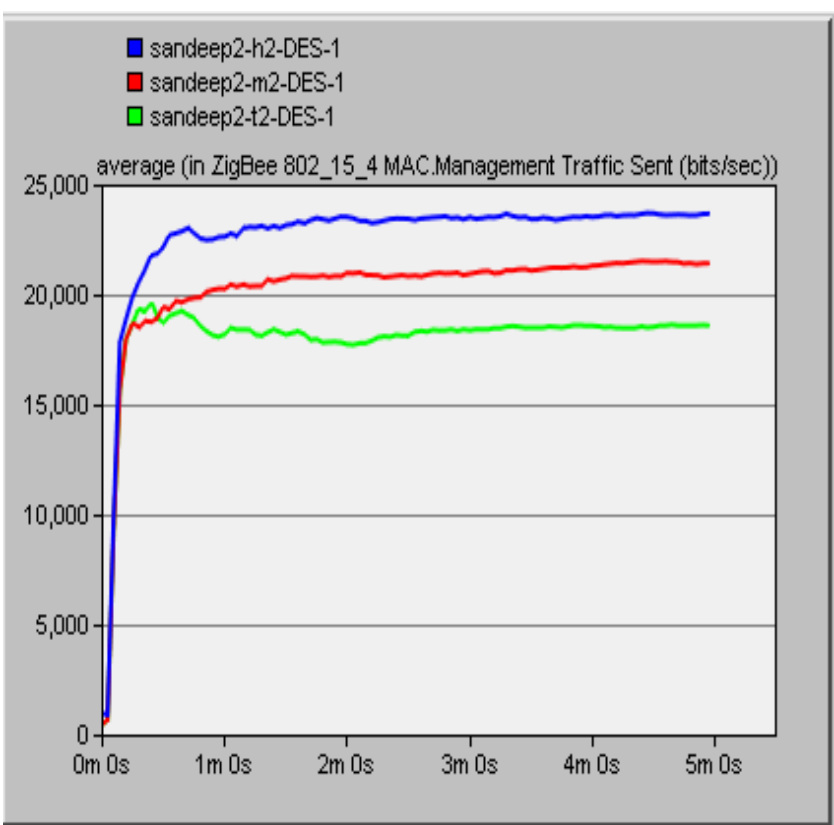

Figure14MTS of tree, mesh and hybrid topologies with $40 \%$ of mobile nodes

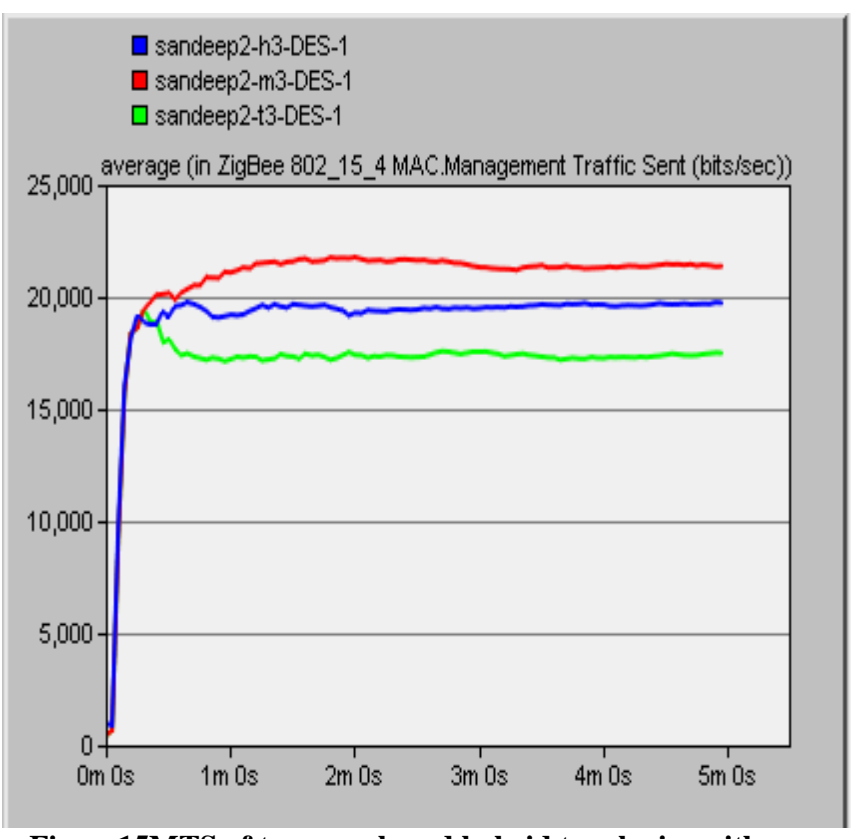

Figure15MTS of tree, mesh and hybrid topologies with $60 \%$ of mobile nodes 


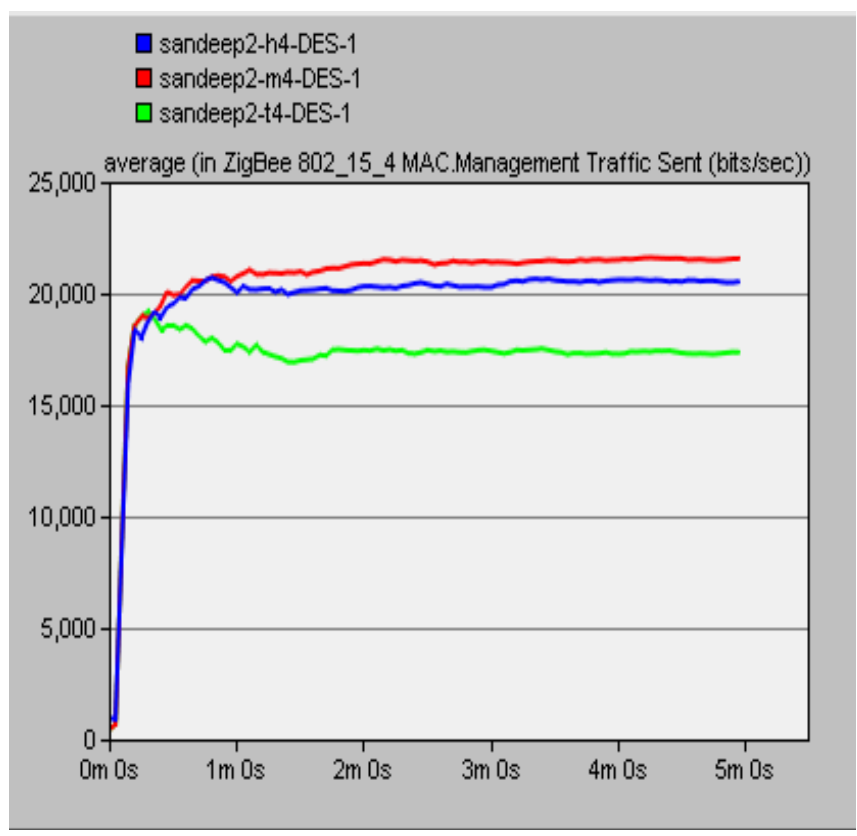

Figure16MTS of tree, mesh and hybrid topologies with $80 \%$ of mobile nodes

\section{CONCLUSION}

This research work show that the tree topology achieved maximum and mesh topology achieved minimum throughput and in case of hybrid topology thethroughput varies as mobile percentage of nodes increases. The hybrid topology offer minimum MAC delay. In the mesh topology MAC delay rapidly increases in the starting and then drop whereas tree topology provides maximum MAC delay. The MTR\& MTSis maximum inmesh topology and tree topology provide minimum MTR \& MTS. Whereas in case of hybrid topology MTR increases in 40 and 80 percent of mobile nodes and decreases in 20 and 60 percent of mobile nodes. MTS in hybrid topology is same in all cases. Hence hybrid topology also shows the effective response in case of mobility.

\section{REFERENCES}
[1] ZigBee Wikipedia.
[2] www.Sena.com

[3] M. Hussnain, M. Sharjeel, S. R. Chaudhry, S. A. Hussain, I. Raza and J. S. Mirza, Investigating MultiTopological ZigBee Based Wireless Sensor Network in Precision Agriculture, ISSN 2090-4304.

[4] Lovish Jaiswal, Jaswinder Kaur, Gurjeevan Singh, "Performance Analysis of Topological Variation in Personal Area Network using ZigBee Wireless Sensors", IJCST Vol. 3, Issue 4, Oct- Dec 2012, ISSN: 22294333(Print)

[5] Ulya Sabeel, Saima Maqbool, Nidhi Chandra, "ZigBee IEEE 802.15.4 Standard for Building Automation", Ijarcsse Volume 3, Issue 6, June 2013, ISSN: 2277 128X.

[6] Manasee Patil, S.R.N. Reddy, "Design and Implementation of Home/Office Automation System based on Wireless Technologies", IJCA Volume 79 No6, October 2013.

[7] Yuan-Yao Shih, Wei-Ho Chung,Pi-Cheng Hsiu, AiChun Pang, "A Mobility-Aware Node Deployment and Tree Construction Framework for ZigBee Wireless Networks", IEEE transaction on vehicular technology, vol. 62, no. 6, July 2013.

[8] Naveen Erasala, David C. Yen," Bluetooth technology: a Strategic analysis of its role in global 3G wireless Communication era “, ELSEVIER, Computer Standards \& Interfaces 24 (2002) 193-206.

[9] Jin-Shyan Lee, Yu-Wei Su, and Chung-Chou Shen, "A Comparative Study of Wireless Protocols: Bluetooth, UWB, ZigBee, and Wi-Fi”, the 33rd Annual Conference of the IEEE Industrial Electronics Society (IECON) Nov. 5-8, 2007, Taipei, Taiwan.

[10] Karunakar Pothuganti1 and Anusha Chitneni,A Comparative Study of Wireless Protocols: Bluetooth, UWB, ZigBee, and Wi-Fi,ISSN 2231-1297, Volume 4, Number 6 (2014), pp. 655-662.

[11] Performance evaluations of ZigBee in different smart grid environments, B.E. Bilgin, V.C. Gungor, Computer Networks 56 (2012) 2196-2205.

[12] Karandeep Malhi, Subhas Chandra Mukhopadhyay, Mathias Haefke, and Hartmut Ewald, "A Zigbee-Based Wearable Physiological Parameters Monitoring System", IEEE sensor journal, vol. 12, NO. 3 march 2012. 